\title{
UTILIZAÇÃO DE FIBRAS DO MESOCARPO E CAROÇO DO AÇAÍ COMO COMPONENTE DE MISTURAS AREIA - ASFALTO PARA A PAVIMENTAÇÃO NA CIDADE DE MANAUS/AM
}

\section{USE OF FIBERS OF THE MESCOPE AND AÇAÍ AS A COMPONENT OF SAND- ASPHALT FOR PAVING IN THE CITY OF MANAUS / AM}

\author{
S. S. dos SANTOS ${ }^{1}$; G.F. da SILVA ${ }^{2,3}$ e D. F. CASTRO ${ }^{1}$ \\ ${ }^{1}$ Faculdade Metropolitana de Manaus - FAMETRO \\ ${ }^{2}$ Universidade do Federal do Amazonas - UFAM, Departamento de Química, Programa de \\ Pós-Graduação em Química \\ ${ }^{3}$ Universidade do Estado do Amazonas - UEA, Escola Superior de Tecnologia \\ E-mail: mollifani@hotmail.com
}

\author{
article info \\ Article history: \\ Received 20 May 2016 \\ Accepted 3 January 2017 \\ Available online 27 July 2017
}

PALAVRAS-CHAVE: Fibras de Açaí; Areia-Asfalto; Ensaio Mecânico; Pavimentação. KEYWORDS: Açai Fibers; Sand-Asphalt; Mechanical Rehearsal; Paving.

\begin{abstract}
RESUMO: A importância das fibras naturais como aditivos ou reforços para produtos poliméricos vem aumentado significativamente nas últimas décadas, devido a fatores como o alto preço das fibras sintéticas e à busca crescente por materiais de baixo custo e que sejam provenientes de fontes renováveis. Ademais devem possuir boas propriedades mecânicas e térmicas, e que não causem danos ambientais. Assim, este trabalho tem como objetivo estudar a utilização das fibras do mesocarpo e caroço do açaí como componente da mistura de areiaasfalto com areias de resíduos de construção e demolição para a pavimentação na Cidade de Manaus-AM. Foram feitas análises termogravimétricas e ensaios mecânicos (DNIT 136/2010ME e ASTM D4123/ AASHTO TP-31-96) bem como a caracterização das fibras e das misturas de areia-asfalto. Por meio desta pesquisa, obteve-se uma maior interação agregado-ligante pelo espesso recobrimento das superfícies dos agregados minerais, devido à absorção de maiores teores de cimento asfáltico de petróleo 50/70. Compósitos reforçados com fibra de açaí in natura apresentaram boas propriedades mecânicas. Portanto, as fibras de açaí, material disponibilizado a custo zero, são passíveis de aplicação como reforço de matrizes cimentícias, proporcionando um bom desempenho mecânico e reduzindo o custo do produto final, devido ao valor das fibras incorrerem somente no seu beneficiamento, que pode ser manual e inserido em contexto social.
\end{abstract}

\footnotetext{
ABSTRACT: The importance of natural fibers as additives or reinforcements for polymer products has increased significantly in recent decades, due to factors such as the high price of synthetic fibers and the increasing search for low-cost materials from renewable sources. In addition they must possess good mechanical and thermal properties, and that do not cause environmental damages. Thus, the objective of this work is to study the use of acai mesocarp and core fibers as a component of sand blasting with sands of construction and demolition waste for paving in the city of Manaus-AM. Thermogravimetric analyzes and mechanical tests (DNIT 136 / 2010ME and ASTM D4123 / AASHTO TP-31-96) were performed as well as the characterization of the fibers and sand-asphalt mixtures. By means of this research, a higher aggregate-binder interaction was obtained by the thick coating of the mineral aggregate surfaces, due to the absorption of higher levels of 50/70 petroleum asphalt cement. Composites reinforced with natural açai fiber presented good mechanical properties. Therefore, açai fibers, available at zero cost, can be applied as reinforcement of cementitious matrices, providing a good mechanical performance and reducing the cost of the final product, due to the value of the fibers only to be processed. Manual and inserted in a social context.
} 


\section{INTRODUÇÃ̃O}

A busca por novos materiais focados na sustentabilidade da Região Amazônica tem sido um crescente na área da pesquisa tecnológica e das Grandes Engenharias. Com isso a importância da utilização das fibras naturais como aditivos ou reforços para produtos poliméricos vem aumentando singularmente nas últimas décadas.

No presente momento estamos vivenciando um déficit na economia do país assim como grandes impactos ambientais, logo existe a necessidade de se obter um material proveniente de fontes renováveis que ajudaria da minimização dos custos e apresentaria propriedades mecânicas e térmicas com potencial para redução dos danos ambientais como, por exemplo, a substituição das fibras sintéticas pelas fibras nativas. Inúmeros estudos têm sido realizados para a utilização destas fibras em indústrias como a de materiais, construção civil, automobilística e aeronáutica.

O açaí, como fruto nativo de nossa Região Amazônica, é um material que apresenta várias propriedades e insumos que podem ser reutilizados como fonte potencial para á área de agregados e ligantes na pavimentação asfáltica.

As principais vantagens da utilização das fibras são: o baixo custo; baixa abrasividade; a toxicidade, baixa densidade; baixo consumo de energia; propriedades mecânicas e termoacústicas adequadas. Quando comparada com as sintéticas incluem também aspectos ecológicos e sociais, em razão da melhor reciclabilidade e biodegrabilidade e do aumento da qualidade de vida dos habitantes de regiões onde é realizado o cultivo (Bledzki; Gassan, 1999; Martins; Joekes, 203).

Levando em consideração a estes inúmeros fatores que agregam valores sócios econômicos e ambientais, este presente trabalho tem por finalidade colaborar nas pesquisas científicas ao estudar a utilização de fibras do açaí como componente de misturas areia-asfalto com areias de resíduos de construção para a pavimentação da Cidade de Manaus-AM.

\subsection{Fibras Ligno-Celulósicas}

As fibras naturais dividem-se em fibras de origem vegetal (fibras de madeira e bambu, sementes, fibras de frutas e fibras de folha), origem mineral (amianto, wollastonita) e de origem animal (fibras de pelo, seda).

As fibras ligno-celulósicas são obtidas dos caules (juta, cânhamo, ráfia,kenaf, bagaço de cana, bambu), das folhas (sisal, caroá, curaua, banana, piaçava, henequem), dos frutos (algodão, coco, bucha) e dos troncos (madeira).

Apesar dessas diferenças, as fibras possuem na sua constituição básica os seguintes componentes em comum: celulose, lignina e polioses, também conhecidas como hemiceluloses. 


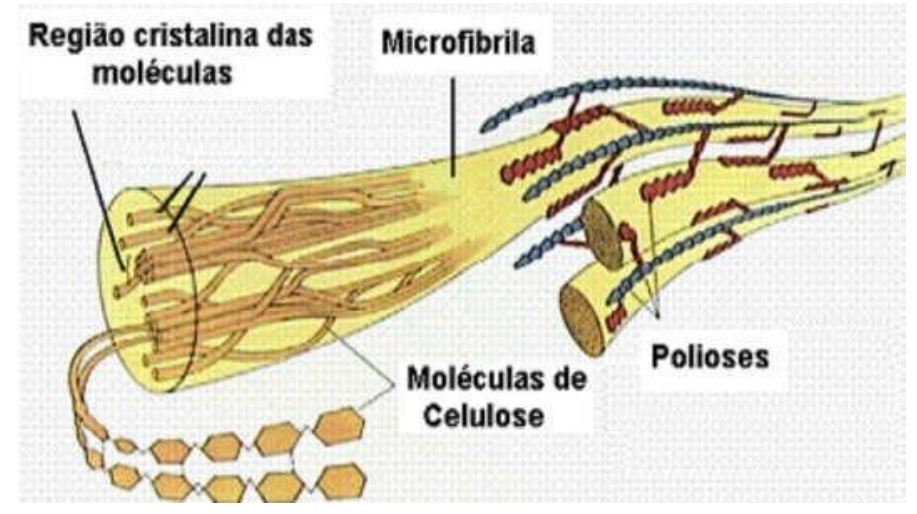

Figura 1 - Estrutura da celulose, esquema da ilustração das moléculas de celulose.

Fisicamente cada fibra ligno-celulósica é composta por quatro partes: parede primária, espessa parede secundária, parede terciária, e o lúmen, como podem se observar na Figura 2. As paredes celulares são formadas de várias camadas de estruturas fibrilares, consistindo de fibrilas.

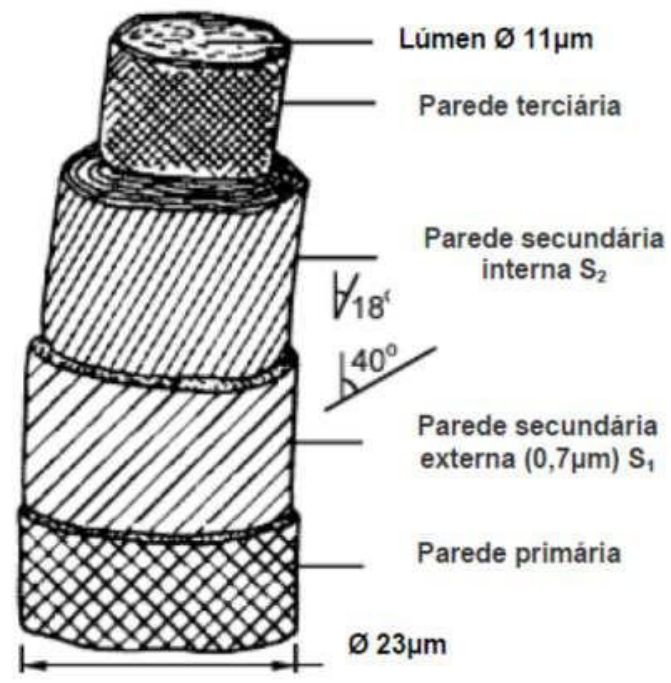

Figura 2 - Estrutura física da fibra ligno-celulósica.

\section{METODOLOGIA}

O presente trabalho foi realizado através de levantamentos bibliográficos, bem como coleta de dados para atender aos objetivos propostos.

\subsection{Caracterização das Fibras de Açaí}

Para a realização da caracterização física do material estudado como agregado, no caso específico a fibra do açaí, realizou-se a separação das fibras do mesocarpo do caroço utilizando-se de uma peneira de pedreiro para obtenção do material desejado. 
Obteve-se por meio de uma trena um comprimento de valor médio de $0,90 \mathrm{~cm}$, o qual pode ser comparado na literatura com um resultado segundo Fagury 2005, que realizou um trabalho similar, porém utilizando-se das fibras nativas do coco (cocus nucifera) com 13,2 $(9,5) \mathrm{cm}$ e da juta $(10 \mathrm{~cm})$.

O material de estudo, fibra do mesocarpo do caroço do açaí, foi escolhido como adicionante à mistura asfáltica para a pavimentação por apresentar em sua estrutura físicoquímica a lignina. De acordo com Passos (2005), a lignina tem a capacidade de ser um ligante muito proveitoso para unir as fibras celulósicas formando a parede celular, fornecendo, por conseguinte, resistência à compressão ao tecido celular e às fibras. Igualmente, sua concentração influencia a estrutura, as propriedades, a morfologia, a flexibilidade e a taxa de hidrólise nas fibras.

Alguns trabalhos já foram publicados utilizando fibras naturais, como o trabalho de Cunha (2011) sobre a castanha de cutia com 33,26\% e as fibras de sisal por Medina (1959) com 9,9\%. Contudo observou-se o resultado satisfatório realizado pela análise de Fagury (2005) que conseguiu obter $11(5,2) \%$ de lignina na fibra do mesocarpo do açaí.

Para a caracterização do analito estudado foi realizada a caracterização térmica das fibras do açaí utilizando as técnicas de termogravimetria (TG) e termogravimetria derivada (DTG). Para a caracterização morfológica foi utilizada a microscopia eletrônica de varredura também conhecida como MEV e microscopia óptica (MO). Os ensaios foram realizados nos laboratórios do Instituto Nacional de Pesquisas da Amazônia - INPA na etapa III.

Ensaios Mecânicos: Para os ensaios mecânicos foram confeccionados os corpos de prova submetidos aos testes de Resistência à Tração (RT) e Módulo de Resiliência (MR) de acordo com a normatização do DNIT (Departamento Nacional de Infraestrutura de Transportes)136/2010-ME e ASTM (American Society for Testing and Materials) D4123/ AASHTO (American Association of State Highway and Transportation Officials) TP-31-96.

Posteriormente foi realizado o Ensaio de Módulo de Resiliência onde os corpos de prova foram submetidos a uma temperatura de $40{ }^{\circ} \mathrm{C}$, temperatura típica da Região Norte do Brasil, e ao carregamento de $5 \%, 10 \%$ e $15 \%$ da maior força normal e de forma axial alcançada por tal mistura no ensaio de RT (Resistência a Tração). Utilizou-se das equações seguintes para se obter os resultados referente a MR e RT.

or $=2 \mathrm{~F} / 100 \pi \mathrm{Dh}$

Onde:

or - Resistência à tração, em MPa

$\mathrm{F}$ - Carga de ruptura, em $\mathrm{N}$

D - Diâmetro do corpo de prova, em cm

$\mathrm{h}$ - Altura do corpo de prova, em $\mathrm{cm}$
$\mathrm{E}=\mathrm{P}(\mathrm{v}+0,2699) \Delta \mathrm{h}$

Onde:

E - Módulo de resiliência, em MPa;

$\mathrm{P}-\%$ da Força axial da resistência à tração, em N;

$\Delta$ - Deslocamento medido, em $\mathrm{mm}$;

$\mathrm{h}$ - Média da altura do corpo de prova, em $\mathrm{mm}$;

$v$ - Coeficiente de Poisson. 


\section{RESULTADOS E DISCUSSÃO}

\subsection{Caracterização Ligante Betuminoso}

A caracterização do ligante betuminoso foi realizada no laboratório da REMAN (Refinaria Isaac Sabbá) utilizando os cimentos asfálticos de petróleo: puro (CAP), modificado com $4 \%$ de polímero EVA (CAP EVA).

Comparando aos resultados das normas da ASTM (the American Society for Testing and Materials) que o cimento asfáltico de acordo com as características de ponto de fulgor, solubilidade, viscosidade, ductilidade e ponto de amolecimento atendem às especificações brasileiras regulamentadas pela Agência Nacional de Petróleo (ANP).

Vale ressaltar que ao se incluir na mistura o copolímero EVA, observou-se uma redução de $69 \%$ para 40,8 \% observando-se que a utilização do adicionante EVA houve uma minimização da Penetração em torno de 40,8 \% e um aumento da Viscosidade de 283 \%para $598,4 \%$, ou seja, tornou o CAP mais consistente.

\subsection{Dosagens}

A dosagem utilizada para este trabalho seguiu os passos da DNIT 032/2005-ES, referente às misturas areia-asfalto AAUQ (Areia Asfáltica Usina a Quente), AAUQ-FIBRA, AAUQEVA, AUUQ-FIBRA-RCD, delimitando-se o teor de ligante betuminoso de $6 \%$ a 12 $\%$.

Em continuação a caracterização e dosagem foram usadas os passos metodológicos para a mistura areia-asfalto de acordo com DNIT.

Entretanto, para as misturas com adicionante da fibra do mesocarpo do caroço do açaí, fez-se a sua incorporação utilizando cerca de $0,5 \%$ do peso total do compósito asfáltico diretamente na fração seca aquecida da mistura, sendo inserida com auxílio de uma peneira e, portanto, antecedente a pesagem do CAP para sua posterior homogeneização.

\subsection{Comportamento Mecânico}

Os corpos de prova foram sujeitados aos ensaios de resistência à tração por compressão diametral (RT) e módulo de resiliência (MR).

No caso do ensaio da Resistência a Tração observa-se o aumento de tal resistência na incorporação do agregado miúdo de RCD, possivelmente justo pela sua contribuição da sua fração cimentícia para a interligação com o agregado-ligante, estudo de misturas areia-asfalto com areia de resíduo de construção e demolição, fibra do açaí e polímeros para a cidade de Manaus, norteando a melhores propriedades mecânicas.

Observa-se também que a sua característica morfológica, apresentando baixa espessura e pequeno comprimento, não atua como elementos de reforço frente às solicitações estáticas, por conseguinte se comportando como uma matriz com vazios, ou seja, gerando um não ajustamento entre o agregado e o material betuminoso. 
No trabalho de Canevarolo (2004), foram registradas as curvas de TG (A) e de termogravimetria e derivada (DTG) das fibras do mesocarpo de açaí em atmosferas de nitrogênio e ar sintético, com taxa de aquecimento de $10{ }^{\circ} \mathrm{C} / \mathrm{min}$.

A Figura 3 apresenta micrografias representativas das fibras que recobrem o caroço do açaí. Por meio desta análise, realizada pelo grupo de pesquisa de geotecnia (GEOTEC-UFAM) pode-se observar deformações irregulares, não podendo ser classificadas como esféricas ou elípticas. Assim, apresenta-se como uma micrografia num aumento maior, onde se podem observar em detalhe os orifícios denominados canais de pontuação presentes em toda a extensão das fibras e células do parênquima que recobrem a superfície.
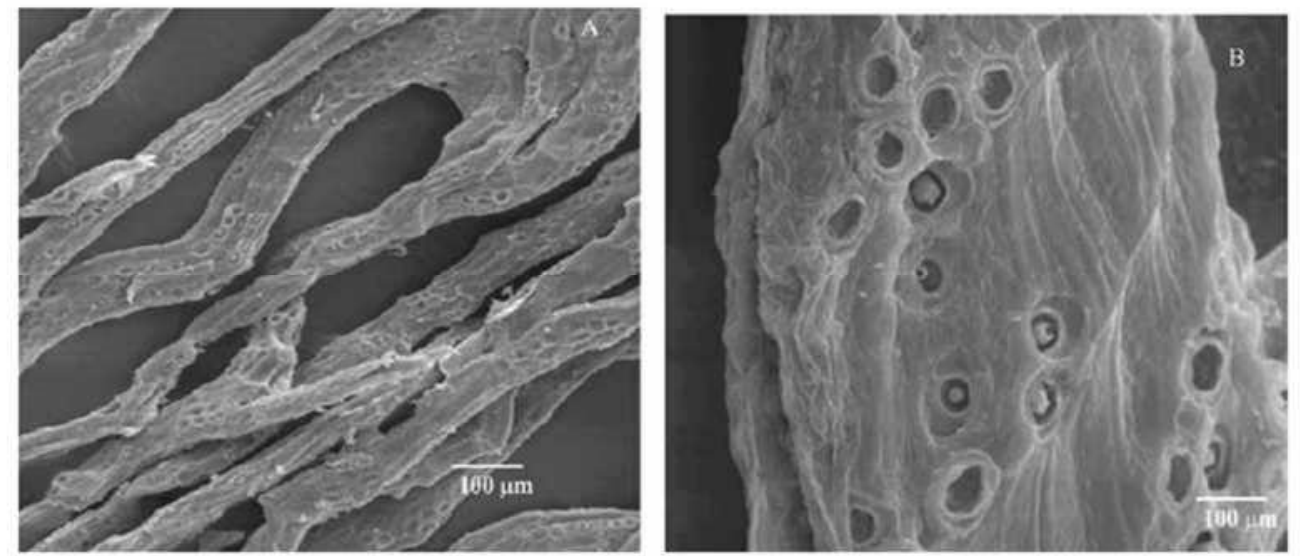

Figura 3 - Micrografias obtidas por MEV da fibra do mesocarpo do açaí.

\section{CONCLUSÕES}

Por meio desta referente pesquisa, pôde-se observar que por meio dos testes de Resistência a Tração (RT) e módulo de resiliência obteve-se um resultado satisfatório com relação ao comportamento físico-químico e mecânico da adição das fibras do mesocarpo do caroço do açaí nas misturas asfálticas.

Observou-se assim a existência de uma maior interação entre as partículas do agregadoligante. Isso ocorreu devido à absorção de maiores teores e concentração de CAP (cimento asfáltico de petróleo) 50/70.

Por mais que exista a necessidade de se obter resultados melhores para esta pesquisa, é importante frisar que trata-se de um material que seria descartado pela sociedade, que por sinal consome bastante este fruto, tornando-se um produto altamente sustentável que acabaria colaborando para um problema econômico, social e principalmente ambiental. 


\section{REFERÊNCIAS}

BLEDZKI, A.K.; GASSAN, J. Composites reinforced with cellulose based fibres. Progress in Polymer Science, Elmsford, v.24, n.2, p.221-274, 1999.

CANEVAROLO Jr., S.V. Técnicas de caracterização de polímeros. São Paulo: Artliber Editora, 2004. 445p.

CUNHA, T. M. F. Estudo do Comportamento Físico, Químico e Reológico de Ligante Asfáltico Modificado com Polímero e Materiais Advindos de Plantas Amazônicas para Aplicação em Revestimentos Asfálticos. 2011. 161 f. Dissertação (Mestrado) Programa de Pós-Graduação em Engenharia de Recursos da Amazônia, Universidade Federal do Amazonas, Manaus, 2011.

FAGURY, R. V. G. Avaliação de fibras naturais para a fabricação de compósitos: Açaí, coco e juta. 2005. 80 f. Dissertação (Mestrado) - Programa de PósGraduação em Engenharia Mecânica, Universidade Federal do Pará, Belém, 2005.

IBGE - Instituto Brasileiro de Geografia e Estatística. Produção da extração vegetal da Silvicultura 2007. Disponível em: Acesso em: 15 de março. 2015.

IBGE. Produção da Extração Vegetal e da Silvicultura. IBGE - Instituto Brasileiro de Geografia e Estatística, v. 24, Brasil, 2009.

MARTINS, M. A.; MATtoso, L. H. C.; PESSOA, J. D. C. Comportamento térmico e caracterização morfológica das fibras de mesocarpo e caroço do açaí. Revista Brasileira de Fruticultura, Jaboticabal, v. 31, n. 4, p. 1150-1157, dez. 2009.

MARTINS, M.A.; JOEKES, I. Tire rubber-sisal composites: effect of mercerization and acetylation on reinforcement. Journal of Applied Polymer Science, Cleveland, v.89, n.9, p.2507-2515, 2003.

MARTINS, M.A.; MATTOSO, L. H.; PESSOA, J.D.C Comportamento Térmico da Fibra de Açaí. São Carlos: EMBRAPA, 2005. 